\title{
EL PSICOANÁLISIS Y SU LUGAR ENTRE LAS CIENCIAS.
}

\author{
PSYCHOANALYSIS AND ITS PLACE AMONG THE SCIENCES.
}

\author{
Juan Pablo Sánchez Domínguez* \\ Universidad Autónoma del Carmen (UNACAR), \\ Ciudad del Carmen - México.
}

Recibido abril de 2018/Received April, 2018

Aceptado agosto de 2018/Accepted August, 2018

\begin{abstract}
RESUMEN
El presente trabajo tiene como propósito identificar el contexto científico que permitió el surgimiento del psicoanálisis, así como las primeras ideas freudianas tendientes a colocar sus descubrimientos al servicio de la ciencia natural, tal y como lo dejó por sentado en el Proyecto de una psicología para neurólogos.

Posteriormente, se definen los desarrollos teóricos y clínicos que le posibilitaron a Freud abandonar su "ideal de cientificidad", al tiempo de construir un método para la investigación de los fenómenos psíquicos inaccesibles por otras vías.

Por último, siguiendo la relectura de Lacan realizada al cogito Cartesiano se especifica la diferencia radical entre el objeto psicoanalítico y el objeto de la ciencia, concluyendo con esto que el psicoanálisis guarda entre las ciencias un lugar extimio.
\end{abstract}

Palabras Clave: Psicoanálisis, Freud, método, Lacan, objeto, ciencia.

\begin{abstract}
The purpose of this paper is to identify the scientific context that allowed the emergence of psychoanalysis, as well as the first Freudian ideas that tended to place their discoveries in the service of natural science, as established in his project Psychology for neurologists.

Later, we define the theoretical and clinical developments that enabled Freud to abandon his "ideal of scientificity" while constructing a method for investigating the psychic phenomena inaccessible through other means.

Finally, according to Lacan's rereading of the Cartesian cogito, we specifiy the radical difference between the psychoanalytical aim and the aim of science, concluding with this that psychoanalysis has an extimacy place among the sciences.
\end{abstract}

Key Words: Psychoanalysis, Freud, method, Lacan, object, science.

\section{Introducción}

Los conocedores de la obra de Freud no pueden negar que su pensamiento estuvo fuertemente influenciado por la racionalidad positivista de su época y por ende sus descubrimientos previos a la formalización del psicoanálisis conservan este matiz, de ahí que sus observaciones clínicas iniciales tengan como base aspectos biológicos (Emilce, 2015). Para Ramírez (2002) y Rudge (2012), a finales del siglo XIX e inicios del XX los hombres de espíritu científico estaban fuertemente encauzados a resguardarse dentro del paradigma positivista, esto como un esfuerzo por oponerse a la metafísica, a las concepciones mágicas y espiritualistas de aquella época. En este contexto,

* Autor correspondiente / Correspondig author: jsanchez@ pampano.unacar.mx 
se pueden identificar en los escritos freudianos iniciales, diversos pasajes cuyo esfuerzo de colocar sus descubrimientos en el campo de las ciencias de la naturaleza era indiscutible (Freud, 1895/1950; Ovejero, 1995; Simanke \& Caropreso, 2005; Emilce, 2015).

Lo expresado por el mismo Freud en 1895 parecía no anticipar el revés que a la postre tendría su pensamiento, al tiempo de darse cuenta de que el conocimiento producido por la ciencia natural resultaba insuficiente para explicar las llamadas -hasta entonces- enfermedades nerviosas. Este descubrimiento le permitirá esbozar sus primeras hipótesis tendientes a establecer un método capaz de tratar y explicar estos fenómenos. Los mismos malestares acaecidos en el cuerpo que le revelaban a la ciencia de su tiempo su límite, le abrían las puertas a la investigación freudiana para la construcción de un saber propio (Rubistein, 1997).

El pensamiento Freudiano elaborado durante 1873 y 1882 que contempla el periodo más fuerte de su formación médica, se vio enormemente influenciado por la fisiología de Brücke llevándolo inclusive a aceptar sin menoscabo que el organismo vivo es un sistema dinámico al que se aplican las leyes de la física y la química (Garcia-Mendes, 1996), de ahí quizás su arraigado interés durante sus primeros esbozos teóricos por conducir sus formulaciones en el marco de una ciencia exacta. Algunos otros vestigios de sus formulaciones en este terreno lo podemos constatar en su escrito sobre la "histeria" de 1888 donde resalta el modo teórico-práctico de su proceder sobre sus pacientes, los cuales no discrepan en absoluto de las "técnicas" empleadas por Charcot y Breuer para el mismo propósito, sin embargo, para 1886, poco tiempo después de concluir su estancia en Paris con Charcot, ya había mostrado señales del camino que posteriormente tomaría llevado por sus teorías clínicas (Lindenmeyer, 2012).

En su artículo publicado en 1893 en los Archives de neurologie se pueden identificar las tesis a las que hasta ese momento había llegado, a saber, que, "la histeria se comporta en su parálisis y en otras manifestaciones como si la anatomía (cerebral) no existiera, o como si no tuviera noticia alguna de esta" (citado por Masson, 1985, p. 9). Para estos años y con base al argumento antes descrito, podemos notar en Freud cierta claridad teórica, sin embargo, su planteamiento metodológico aún no contaba con la evidencia clínica suficiente como para distanciarse de las técnicas habituales empleadas por la medicina para el tratamiento de los padecimientos histéricos, tal es el caso que en las primeras comunicaciones firmadas con Breuer, Freud aún realiza el abordaje de sus pacientes confiando en las técnicas terapéuticas de antaño: hidroterapia, electroterapia, etc., en este mismo sentido, mostrará un claro optimismo al profesar en una carta dirigida a Fliess lo siguiente: "he emprendido la hipnosis y he tenido toda suerte de pequeños pero notables éxitos" (Breuer \& Freud, 1893-95/1992).

Contrariamente, mientras transcurría el año de 1895 Freud escribía un texto que a posteriori se tornaría por demás paradigmático en la historia del movimiento psicoanalítico, a saber, el "Proyecto de una Psicología para neurólogos". Este documento contiene las ideas prínceps que dieron lugar a toda la formulación posterior en torno al "funcionamiento psíquico" también representa el desvanecimiento de los intentos freudianos por hacer que sus aportaciones sobre los malestares subjetivos se incluyeran en los anales de las ciencias duras (Fulgencio, 2004; Henríquez, 2010).

Al hacer una lectura del "Proyecto de una Psicología para neurólogos" es evidente que el escrito en su conjunto está plagado de metáforas mecanicistas y de un vocabulario propio de las ciencias físicas y biológicas (Antonello \& Oliveira, 2011), sin embargo, coincidiendo con Ramírez (2002), planteamos que este modo de escritura no menoscaba su valor en lo concerniente a la importancia de las hipótesis ahí sostenidas, al final de la cuestión, las ideas elaboradas dentro del "Proyecto" le posibilitaron a Freud comprobar que el aparato biológico no le permitiría dilucidar de ningún modo la propiedad de ser o no consiente, este descubrimiento favoreció enormemente su teoría de la necesidad de afianzar un método que permitiera la investigación de los fenómenos histéricos por otros medios que no fueran los hasta entonces empleados. En este contexto, Freud se vio conducido a apartarse de los procedimientos utilizados por la ciencia médica a fin de avanzar en su nuevo propósito (Freud, 1893-99/1992), sin embargo, en 1924 admitirá lo siguiente:

(...) era preciso extraer del hipnotismo dos doctrinas fundamentales e inolvidables. En primer lugar, se llegó al convencimiento de 
que aun alteraciones corporales llamativas podían ser el resultado de influjos puramente anímicos, activados por el experimentador mismo; en segundo lugar, y en particular a raíz de la conducta de los sujetos tras la hipnosis, se tuvo la impresión más nítida de la existencia de procesos anímicos a los que no se podía dar otro nombre que el de «Inconscientes». (Freud, 1923-25/1992, p. 204).

Esta formulación construida a posteriori por Freud sintetiza que sus hallazgos fueron forjados en el caldero de los hechos reales de cada paciente, demostrables y colegibles bajo cierta condición clínica, teórica y metodológica, si bien, para la ciencia positiva de su época estos descubrimientos carecían de validez, la verdad que trajo consigo para la comprensión de la realidad humana fue históricamente irreversible. El efecto de rechazo por parte de los círculos científicos de su tiempo sobre los postulados freudianos que advertían la existencia de procesos anímicos inconscientes encuentra sentido al momento de reconocer que la "ciencia natural" durante largos años se había conformado con una explicación de la realidad humana enmarcada en la experiencia temporal de la conciencia (Coccoz, 2012; Tubert, 2007).

\section{Contexto positivista y objetividad científica}

La obra titulada "El discurso del método" escrita por Rene Descartes durante el siglo XVII sentó las bases de aquello que más tarde recibiría el nombre de ciencia moderna (Harada, 2006; González, Domínguez, Fabre, \& Cubero, 2010; Berrios, 2011; Boonen \& Calazans, 2017). El establecimiento de esta trajo como resultado la hipótesis de que, para construir un saber verdaderamente científico sobre los fenómenos acaecidos en el mundo, era necesario mantener el carácter de objetividad en su abordaje (Méndez, 2000). Es preciso reconocer que la propuesta Cartesiana del cogito también favoreció el progreso de un pensamiento que a la postre lograría arrebatarle al discurso religioso el criterio de verdad sobre los objetos. Para Brousse (2012), el cogito Cartesiano fue la propuesta que permitió dar el paso decisivo para colocar la racionalidad científica por encima del dogmatismo religioso, el mismo que había fungido durante la Edad Media como la forma categórica para explicar todos los fenómenos. Sobre estas coordenadas la llamada ciencia moderna floreció (Lacan, 1975/2011), al tiempo en que se posicionaba la propuesta del cogito Cartesiano como la fórmula de toda representación del sujeto dentro del pensamiento occidental, aludiendo al propósito de encontrar un pilar indubitable que diera paso a la construcción de un saber sobre los objetos.

Con el tiempo y bajo este lógica, la investigación científica logró hacer del pensamiento la evidencia de la existencia de los objetos, al precio de reducir la subjetividad a una racionalidad vacía de representación, conducente a una especie de mentalismo ensimismado (Brousse, 2012). En otras palabras, el cogito Cartesiano permitió sentar las bases de un conocimiento necesitado por la ciencia y que a la larga lograría cercar la cuestión de la verdad en relación con la constitución de los saberes, no obstante, el precio que tuvo que pagar fue despojar a este nuevo sujeto de todas sus pasiones, es decir de sus atributos subjetivos.

Con la tesis propuesta por Descartes acerca de la existencia de una sola verdad para cada cosa, la construcción de un saber sobre los objetos se redujo a la puesta en marcha de un método, y éste como la vía más sólida del fundamento mismo de la ciencia (Méndez, 2000). A partir de ello, la lógica se asentará en un racionalismo dual, por un lado, un sujeto que piensa y por el otro, un objeto que puede ser pensado. Para Jardim y Rojas (2010), esta fórmula cognoscente y racional sobre el mundo, conducirá a la ciencia de la época a la creación de mecanismos tendientes a conservar su radical materialidad; bajo la clara separación entre el sujeto y el objeto se construirá el ideal de objetividad científica (González et al., 2014), al costo de atenerse casi en exclusividad a estudios de carácter "fáctico" y de explicaciones de tipo causalista (Sánchez, Sánchez \& Sánchez, 2005).

Para González et al. (2010) la física Aristotélica fue el antecedente racional del método científico tal y como lo propuso Descartes, este a su vez impulsó el paradigma positivista que a finales del siglo XVIII y principios del XIX le daría a la ciencia natural el marco perfecto para legitimarse como el modo único de construir un saber sobre los fenómenos del mundo, por medio de la experiencia rigurosamente controlada. En otras palabras, para el positivismo, la única forma de construir un saber sistematizado fuera de toda especulación es atendiendo mediante procedimientos confiables a la neutralidad valorativa del investigador, al momento de abordar el objeto que 
estudia. La premisa que sostiene este postulado es la que señala que entre sujeto-objeto es posible una separación simple y radical, como si entre estos elementos no operara ninguna relación de mediación (Schanzer \& Wheeler, 2010). Al respecto, Daston y Galison (2007) sostienen que esta definición de objetividad dentro de las ciencias positivas no es más que una visión confusa de la realidad, que sitúa a quien investiga en una posición deliberadamente cegada, que le obliga a colocarse frente al objeto en una perspectiva mecanizada. Otros autores han criticado fuertemente esta racionalidad al determinar que este ideal de objetividad científica sigue siendo aún impreciso y reposa en presupuestos enteramente cuestionables, provenientes de teorías psicológicas poco rigurosas (Cupani, 2011).

Por otro lado, Harada (2006) y Cupani (2011) refieren que el progreso del conocimiento sobre la realidad humana solo es posible en la medida en que las disciplinas dedicadas a su estudio renuncien al ideal de objetividad y neutralidad, por su parte Chavarría (2011) será más radical al señalar que si en el campo de la subjetividad humana aceptamos esta apreciación positivista sobre objetividad, estaríamos condenados sin duda, al fracaso. En el marco de estas reflexiones se puede señalar que el criterio de objetividad adoptado por las ciencias positivistas ha permanecido condicionado por ideologías sociales, políticas y económicas según cada momento histórico.

Dicho lo anterior, el discurso científico de la época que a Freud le tocó vivir estuvo enmarcado en los triunfos de la racionalidad positivista, tal paradigma había florecido como una alternativa para abordar de manera metodológica los hechos en su carácter autónomo y de este modo alejarse de la racionalidad espiritualista con las que el romanticismo había embrujado a las personalidades de inclinación científica de ese tiempo (Jiménez, 1994; Ramírez, 2002; Gargiulo, 2014). Esta doctrina sostenía que la única vía para producir un conocimiento objetivo era dando respuesta a hipótesis mediante procedimientos empíricos cuidadosamente observados, controlados y verificados (Pezo, 2006; Villacañas, 2011; França, 2016), el éxito de esta corriente fue tal al grado de asentarse la idea de que este método podría trasladarse sin modificación a otras áreas del conocimiento dedicadas al abordaje de fenómenos no objetivables (Coccoz, 2012). Fue así que para finales del siglo XIX, al menos en Europa, los estudios orientados a resolver los problemas sociales y humanos estuvieron fuertemente influenciados y realizados bajo un notable matiz positivista (Jiménez, 1994). En este tenor, Rodríguez en 2010, señala que las ciencias sociales en su afán por distanciarse del pensamiento mítico religioso y de la subjetividad propia de los procesos humanos, estuvieron desde sus inicios orientadas a solicitar cobijo del naciente paradigma positivista, aunado a esto y en sus aspiraciones por procedimientos con validez científica éstas cayeron en el error de un excesivo metodologismo, provocando con ello la separación de las cualidades de su objeto de estudio.

El resultado antes descrito fue lo que Freud superó no sin la preocupación de separarse de esta doctrina con la que durante largos años mantuvo una interlocución constante, producto de sus intentos por legitimar sus descubrimientos bajo tales reglas. Una vez socavados sus anhelos de objetividad científica, Freud insistirá en señalar que sus hipótesis no son resultado de simples especulaciones, por el contrario, surgen de una laboriosa investigación de cada enfermo y está conducida bajo una extrema minuciosidad (Freud, 1896-99/1992). En otras palabras,

Los conceptos freudianos se validan tanto por una necesidad lógica que los legitima al hacer inteligible un campo de fenómenos de otro modo inexplicable, como por las pruebas clínicas aportadas por la investigación realizada con el método analítico. (Rubistein, 1997).

En resumen, el encuentro con lo contingente de sus pacientes le indicó a Freud el camino a seguir para el estudio de la subjetividad, investigación que le permitiría formular un cuerpo teórico riguroso y con el tiempo la formalización de un método capaz de estudiar los procesos psíquicos inasequibles por otras vías.

Para autores como Lacan, (1964/2009); Mannoni, (2002); Miller, (2011), entre otros, el inventor del psicoanálisis va a localizar muy tempranamente en el discurso de sus histéricas un "saber" qué incluso para ellas permanecía fuera de su propio dominio, aunque el descubrimiento de este saber no se lo podemos atribuir a Freud, sí su modo de abordarlo. Antes de la invención del psicoanálisis este saber no era interés de las disciplinas bio-médicas, contrariamente, se había 
convertido para ellas en una dificultad teóricometodológica (Canale, 2011). A todo pasado, podemos señalar que este obstáculo científico atizó el surgimiento del psicoanálisis, es decir que, allí donde la medicina afrontó el problema creando nuevos mecanismos orientados a separar del mundo a estos sujetos mediante su aislamiento en los espacios hospitalarios, Freud fundó la "cura por la palabra" (Gammelgaard, 2015).

Sabemos históricamente que los hospitales fueron el escenario perfecto para la exclusión de las histéricas, sin embargo y paradójicamente estas mismas "enfermas" se encargarían de cautivar el interés de Martín Charcot, uno de los neurólogos más prominentes de la época. Este médico parisino gracias a la aplicación de la hipnosis logró demostrar la existencia de leyes "no orgánicas" que operaban sobre la producción de fenómenos histéricos (Mannoni, 2002; Rancaño, 2011) pero, coincidiendo con su racionalidad biológica presentará su descubrimiento como una deficiencia científica, a falta de una explicación por la vía del órgano de estos malestares (Canale, 2011). Será este el modo en que se rechace, por parte de la ciencia llamada natural las primeras manifestaciones clínicas de un saber subjetivo, contrariamente, el camino que se tomará será la elaboración de cuadros descriptivos que como dato permitirán posteriormente la elaboración de una clasificación diagnóstica (Sánchez, 2016a). Para González, Hernández y Antonio (2014), este modo de proceder llevaba a cuesta la influencia de una doctrina positiva que se planteaba en ese entonces como la única alternativa para la producción de un conocimiento sistematizado sobre los objetos, fueran estos naturales o no, de ahí que la ciencia médica en general asumiera sin titubeos mantenerse en el ideal de objetividad propuesto por este paradigma, formulándose a posteriori como el único discurso autorizado para construir un saber "exacto y verdadero" sobre los malestares acaecidos en el cuerpo.

\section{Del proyecto freudiano}

Como lo señalamos con anterioridad, Freud como médico no fue ajeno al espíritu positivista de su época, al menos así lo dejo por sentado "parcialmente" en 1895, año en que escribió uno de los documentos de mayor envergadura para la germinación de sus ideas, mismo que posteriormente a su deceso se publicara con el nombre de "Proyecto de una psicología para neurólogos".

El trabajo emprendido en esta empresa no tendrá los resultados científicos a los cuales Freud aspiraba, a pesar del empeño depositado a esta labor.

Tomando como referencia la correspondencia completa de S. Freud a W. Fliess publicada por Masson en 1985 y que constituye un testimonio de la prehistoria del psicoanálisis (Fucks, 2017), podemos dar prueba de la dedicación que el psicoanalista Vienés depositó durante la gestación de este escrito, tenemos que, en una carta fechada el 27 de abril de 1895 manifestó sentir cierta obstinación sobre la tarea de una psicología para neurólogos, a tal grado de consagrarle todo su tiempo; un mes después de estas declaraciones le dará a este trabajo el estatuto de ser su "pasión dominante" y el cual, según él, veía cada día más cerca de concluirse exitosamente, sin embargo estas expectativas sucumbieron pronto. En su carta del 16 de agosto de ese mismo año afirmará que esa psicología es realmente un calvario; por último, en su correspondencia del 8 de noviembre expresará la idea de mandar el documento a dormir a un cajón, no obstante, para enero de 1896 le entregará a Fliess algunas de las elaboraciones fundamentales adoptadas en el proyecto referido.

El documento enviado a Fliess permanecerá en el olvido hasta 1936, en ese año y en el contexto de la comunicación que Marie Bonaparte ${ }^{1}$ sostenía con Freud, esta le expresará lo siguiente: "Hoy se me presentó un señor Stahl de Berlín. Ha obtenido de la viuda de Fliess las cartas y manuscritos de usted" (Masson, 1985, p. 20), tiempo después Freud refiriéndose a este documento contestará lo siguiente, “(...) No me gustaría que nada de esto llegara a conocimiento de la llamada posteridad (...)" (Masson, 1985, p. 21).

En el contexto señalado, la negativa de Freud por hacer público sus ideas contenidas en el "Proyecto (...)" toma sentido si consideramos el "impasse"2 que él produce al tiempo de poner en manos de Fliess un documento que hoy sabemos permaneció olvidado durante largos años. Para Lutereau (2010), una de las causas que llevaron al abandono de este monumental libro está fuertemente ligada a los propósitos inalcanzados por parte de Freud para introducir la "psicología" a través de una puerta tan estrecha como lo son las ciencias naturales. Sin embargo, las directrices 
formales de las ideas contenidas en esta empresa no dejaran de estar presentes sobre todo en su interés geniuno por explicar satisfactoriamente los fenómenos clínicos revelados en su práctica. Si bien estos hechos a la luz del "Proyecto (...)" buscaban encarecidamente una explicación biológica, como resultado del tratamiento de sus histéricas su mirada será empujada a prestar mayor atención a su escucha clínica. En este camino Freud se fue sobreponiendo no sin altibajos a una serie de contratiempos mostrados en su "Proyecto (...)" el estudio sobre "La interpretación de los sueños" le rendía frutos liberando sus postulados de cierto peso cuantitativo y neurológico. Esta propuesta denominada "metapsicológica" proponía una nueva explicación soportada en una estructura de representaciones.

Al tratarse de una serie de ideas que no se sostenían en una representación real sino en la constatación de una operación subjetiva, este nuevo modelo presentaba su propio obstáculo, este escollo deslizaba la labor de Freud cada vez más fuera de los cánones de cualquier ciencia natural. El inconveniente teórico será resuelto más tarde con el desarrollo y esclarecimiento del punto de vista económico, según Lutereau (2010) fue gracias a que en dicho punto de vista Freud se dedicó a ponderar la función de la experiencia de satisfacción sobre el objeto.

Dicho lo anterior, a pesar de las negativas expresadas por Freud de que las ideas contenidas en su "Proyecto (...)" no vieran la luz pública, los postulados ahí vertidos se convirtieron claramente en una especie de matriz de trabajo al menos hasta la elaboración de los "Trabajos sobre metapsicología" establecidos alrededor de 1915.

\section{Hacia una investigación en psicoanálisis}

Las ciencias enmarcadas en una tradición positivista pretenden mantener clausurada la manifestación subjetiva del investigador al tiempo de abordar el objeto que estudia, esto mediante una estrategia racional y normalizada que posibilite salvaguardar al sujeto de cualquier tipo de apreciación singular como resultado de esta interacción (Brooks, 2017). Este aparato científico pretende bajo el marco de la neutralidad, hacer de la experiencia humana de investigar un proceso objetivo, controlable, repetible y comprobable; contrario a esta rigidez racional el psicoanálisis nos ha demostrado que, la realidad de quien investiga no puede desarticularse de los procesos de subjetivación que constituyen su posición misma como sujetos deseosos de producir un saber.

Freud desde los inicios de su investigación clínica planteó la existencia indisociable entre él y sus pacientes, inclusive, refiere haber valorado en sus indagaciones observaciones sobre sí mismo (Masson, 1985, p. 545). Freud a lo largo de toda su obra formuló de manera insistente un conjunto de reflexiones y discusiones con el propósito de otorgarle un cuerpo teórico al efecto derivado de la relación médico-paciente, refirió que era imposible deslindarse de sus efectos al tiempo de ponerse en marcha la pesquisa del inconsciente (Barcellos \& Magdaleno, 2012). Esta relación que más tarde establecería como transferencia tiene por cualidad escapar a cualquier operación racional. El inventor del psicoanálisis será radical al proponer a la transferencia dentro de la investigación psicoanalítica como el punto nodal para poner en acto el saber inconsciente y acceder a él (Ávila, 2005; Sánchez, 2016b).

Empujado por el desafió que le imponía su disciplina en la generación de evidencias que se tradujeran posteriormente en nociones diagnósticas, Charcot mediante la hipótesis de una escena traumática y apoyándose en la técnica hipnótica provocará episodios demostrativos en sus pacientes. Sobrevalorando su interés científico por encima del sufrimiento de sus enfermas y desestimando por completo la elaboración subjetiva que en todo caso había movilizado la relación terapéutica, el médico parisino dejará de escuchar aquello que más tarde le abrirá a Freud las puertas para la formalización de su método.

\section{Un día en este acto de demostración Charcot produjo una contractura de lengua y laringe (...) como consecuencia la paciente afónica sufre calambres en el cuello, al mismo tiempo que el juego teatral se internaliza, cuando recupera el habla la paciente exclama: 'me dijiste que me curarías, que me harías diferente. Tú querías que yo fracasara, me sonsacas lo que quieres saber'. (Mannoni, 2002, p. 19, [entrecomillado propio]).}

En palabras de la paciente se puede entrever el proceder de una ciencia médica que con el firme propósito de producir un conocimiento sobre el objeto que estudia, pierde de vista las cualidades de este, socavando toda posible formulación alrededor 
de la relación subjetiva entre el sujeto que investiga y el objeto investigado. Esta demanda de la paciente a Charcot representa aquello que Freud supo tempranamente sospechar, a decir qué, el saber del médico queda estocado cuando se trata de las singularidades de las histéricas. En otras palabras, lo que el caso sintetiza es una racionalidad medico científica que no logró producir un saber nuevo sobre los cuerpos como efecto de su intervención y que paradójicamente al tiempo en que Freud se fue alejando de este conocimiento previamente configurado, sentaba las bases del psicoanálisis (Gildersleeve, 2016).

En la obra establecida por Breuer y Freud (1893-95/1992) se identifica el reconocimiento que estos realizan a Pierre Janet ${ }^{3}$ por su destacada investigación en torno a las consecuencias terapéuticas derivadas de la relación médico-paciente, sin embargo, también se puede notar que la manera de afrontarlo no distó de la posición científica sostenida por Jean-Martin Charcot.

Lo que hemos descrito como la emergencia de un "saber subjetivo" derivado de la relación sujeto-objeto provocado por la ciencia médica pero no previsto ni asumido por ella será para Legendre (2008) el "accidente científico" que de paso a los esquemas teórico-prácticos que posteriormente le permitieron a Freud formular una nueva disciplina alejada de dos racionalidades contemperantemente posibles, la primera proveniente de un paradigma positivista dominante y la segunda derivada de una visión metafísica del mundo que resistía su decadencia. Con el paso del tiempo y asumiendo el desafío que representaba para él la explicación de los fenómenos acaecidos en cada paciente, alrededor del año de 1895 decidirá iniciar sus indagaciones mediante la técnica de las "asociaciones libres" abandonando cada vez más la mecánica de la sugestión deliberada (Freud, 1893-99/1992). Esta operación clínica consiste básicamente en invitar al paciente a decir todo lo que se le ocurra sin censurar nada, dichas producciones son interpretadas a decir de Freud únicamente en el contexto del tratamiento y bajo la lógica de la transferencia, años más tarde declarará que en términos metodológicos todo aquel que decida sumarse a la investigación de los fenómenos psíquicos se verá necesitado de encarar dicha práctica con la asimilación de su propio análisis (Freud, 1915-16/1992).
Lo anterior permite precisar que dentro del campo de la investigación psicoanalítica, más allá de plantearse cierto neutralismo controlable por medio de un procedimiento normativo y externo, se apela a la tesis de que el sujeto que emprenda el camino de la investigación en este terreno experimente los efectos propios de su análisis, con el firme propósito de que sus intervenciones no se vean socavadas por sus síntomas.

Dicho esto, el análisis personal hace posible que el investigador se interrogue sobre las dificultades que se le presentan en su práctica clínica-investigativa, en otras palabras, no basta con intervenir, es preciso saber sobre los efectos de esas intervenciones. A modo de ejemplificar lo expuesto tenemos que, en el caso "Dora" publicado por Freud en 1905 el padre del psicoanálisis logró precisar y traer a la luz las dificultades transferenciales que durante el abordaje de la paciente se le presentaron. Con posterioridad propondrá que las proyecciones que se desplegaron durante el tratamiento estuvieron motivadas por la relación personal establecida con ella, encontrando ahí la estructura común que encierra esta relación, sin embargo, concluirá también que gracias a este vínculo se genera una diferencia radical que coloca al analista en el lugar privilegiado de "sujeto supuesto saber" y del cual en el análisis se podrá sacar provecho, a condición de que su deseo de saber no esté dirigido a convertirse en soporte de las identificaciones de aquello que provoca (Sánchez2016a).

\section{Los pasos de Freud}

Una vez identificado los aspectos subjetivos implicados en el acto de investigar los fenómenos histéricos y su respectivo rechazo por la ciencia natural, el inventor del psicoanálisis describirá que difícilmente se puede explicar el comportamiento humano en términos mecánicos. En los primeros días del siglo XX en una redacción dirigida a su amigo Fliess se negará a ser llamado "hombre de ciencia" (Masson, 1985, p. 436).

La llegada del nuevo siglo también conformaría el marco perfecto para la presentación en público de quizás, una de las formulaciones freudianas de mayor envergadura, a saber, que gracias a los sueños consiguió aclarar los mecanismos de las formaciones del inconsciente (Torrades, 2005; Rudge, 2016). Tal era la importancia de esta obra que él mismo dejó por sentado que a pesar 
de haberla escrito en el invierno de 1899 su libro "La interpretación de los sueños, fue posdatado para que apareciera como parte del nuevo siglo (Freud, 1900/1992, p. 5).

Las elaboraciones realizadas en este documento traerán consigo efectos radicales, por un lado, Freud llegará a formalizar su lucha por desprenderse de la "racionalidad objetivista" vigente para la ciencia de su época (Ruiz, 2009) y por el otro, se intensificará su crítica sobre la desestimación de otros fenómenos subjetivos como: el chiste, los síntomas, los lapsus, etcétera, en el supuesto libre albedrío del yo al momento de hacer elecciones conscientes (Blas, Azcona \& Ortiz, 2013).

En palabras del autor y refiriéndose al logro conseguido en la "Interpretación de los sueños" comenta qué, el resultado en esta empresa no habría sido posible si la ciencia natural no hubiera negado a este objeto, su valor fundamental (Freud, 1900/1992). Tiempo después precisará las causas de su movimiento expresando que el propósito de su método no es dejar fuera el estado convencional de la medicina, sino poner en marcha un dispositivo donde los fenómenos subjetivos sean tomados en cuenta (Freud, 1906-08/1992).

A posteriori de una relectura de la obra psicoanalítica se pueden reconocer los esfuerzos por parte de Freud por sumarse a los ideales de cientificidad natural, no obstante, el camino que le permitió "la interpretación de los sueños" le condujo a precisar, criticar e interrogar a esta (Emilce, 2015). Las consecuencias por construir una nueva causalidad acerca de la realidad humana se tradujeron también en el más severo repudio por parte de aquellos defensores del ideal de objetividad científica (Prudente \& Ribeiro, 2005).

Dicho lo anterior y varios años más tarde en una publicación realizada bajo el título "Contribuciones a la historia del movimiento psicoanalítico (...)", publicará lo siguiente:

yo trataba mis descubrimientos como contribuciones a la ciencia y lo mismo esperaba que hicieran los otros (...), el vacío que se hizo en torno a mi persona, las insinuaciones que me fueron llegando, me hicieron comprender poco a poco que mi tesis... no podía tener la misma aceptación. (Freud, 1914-16/1992, p. 20).
En el marco de estas reflexiones Freud también tildará a la investigación psiquiátrica de haberse conformado únicamente con describir las perturbaciones del alma, y que estas al no ser llevadas a los terrenos de la singularidad le ha traído severas consecuencias para la generación de evidencia sobre la etiología y mecanismos de formación de síntomas. Contrariando la práctica, señala que gracias a que el psicoanálisis asumió desde el principio la importancia de los fenómenos anímicos presentes en cada paciente esta ha logrado una estructura y formalización que ninguna otra disciplina ha podido alcanzar a pesar de los años (Freud, 1915-16/1992). Manteniéndose firme a las declaraciones de su escrito "De la historia de una neurosis infantil (...)" será más agudo al declarar que, si la medicina no asume la importancia que poseen los elementos psíquicos en la investigación de la enfermedad y su tratamiento, esto le conducirá a una deficiencia evidentemente práctica (Freud, 1917-19/1992).

Para 1920, año en que será publicado su trabajo acerca del "Más allá de principio de placer (...)" volverá a situar el menosprecio que se había ganado dentro del campo médico al tiempo de que sus descubrimientos encontraban camino fuera de las ciencias duras, citamos:

\begin{abstract}
No parece nuestro destino trabajar en paz en la construcción del psicoanálisis. Apenas acabamos de rechazar con éxito dos ataques, uno pretendía desmentir todo cuento hemos traído a la luz (...) el otro, quería persuadirnos de que habíamos equivocado el origen de ese contenido y debíamos cambiarlo a la ligera por otro, 'apenas, entonces, acabamos de sentirnos a salvo de estos enemigos' y ya se eleva frente a nosotros un peligro nuevo. (Freud, 1920-22/1992, p. 169).
\end{abstract}

Para Pezo (2006), todas estas manifestaciones de confrontación realizadas al inventor del psicoanálisis son correlativas, por un lado, con una "ciencia natural" que apoyada de un paradigma positivista estaba de acuerdo con desmentir el saber producido por este naciente método y, por otro lado, la consecuencia de la posición teórica asumida por Freud a partir de sus descubrimientos y que lo llevaron finalmente a una renuncia necesaria a sus aspiraciones científicas iniciales (Prudente \& Ribeiro, 2005). 
Algunos autores coinciden en afirmar lo difícil que sigue siendo para el psicoanálisis dejar atrás el "fantasma" que atravesaron las aspiraciones científicas freudianas contenidas en el "Proyecto (...)" incluso, a pesar de todas las evidencias por parte de Freud por delimitar y legitimar su validez teórica y metodológica. Habermas (1968/1982) después de haber realizado una lectura a la obra Freudiana concluirá que esta invención representa una forma de saber privilegiado fuera de todo cientificismo. De igual modo, autores más contemporáneos (Gutiérrez, 2004) serán más estrictos al respecto, proponiendo que los reproches atribuidos al psicoanálisis en lo que respecta a su "veracidad y objetividad" aún conservan los vestigios de un espinoso descontento gestado en las filas del paradigma positivista; los autores también reconocen que estos juicios acerca de su validez han estado orientados por modelos incompatibles con el objeto de estudio psicoanalítico.

En suma, Jardim y Rojas (2010), refieren que generalmente las críticas realizadas tanto a Freud como a su dispositivo parten de la falsa idea de que el psicoanálisis mismo se postula como un "quehacer científico" de índole experimental.

\section{El psicoanálisis frente al objetivismo científico}

Como lo hemos señalado anticipadamente, el dialogo que Freud sostuvo con la ciencia positiva de su época fue frecuente como también lo fue su crítica hacia ella, esto le condujo finalmente al abandono de su ideal de objetividad.

En una obra publicada un año posterior a su fallecimiento reconoció nuevamente sus intentos iniciales de poner sus hallazgos psíquicos al servicio de la ciencia llamada exacta, sin embargo, estos no encontraron comprensión alguna, peor aún -remarca- encontraron una "laguna" (Freud, 1937-39/1992).

A todo pasado se puede apreciar el proceder ético de Freud durante la construcción del psicoanálisis, en otras palabras, a pesar de los conflictos internos y las discordancias teórico-metodológicos que le traía consigo la publicación de sus resultados tal y como los encontraba en su práctica jamás dudo en hacerlas públicas, al costo de retrocesos en sus hipótesis y confrontar a todo un cuerpo ideológico que dominaba el aparto científico de su tiempo.
Con el paso de los años la causa freudiana rendiría frutos al establecerse como un dispositivo de investigación capaz de abordar los aspectos subjetivos inaccesibles por otros campos del saber (Armengol, 2007; Tessier, 2012; Sánchez, 2016a), llegando incluso a establecer la tesis de que en el acto de investigar se produce entre sujeto-objeto un efecto irreductible al control mediante procedimientos racionales.

A pesar de las batallas que el inventor del psicoanálisis afrontó a fin de otorgarle un claro distanciamiento a su dispositivo de investigación de las esferas de la objetividad positiva, esto no fue suficiente para resolver de fondo el problema, paradójicamente años más tarde Jacques Marie Lacan $^{4}$ sería llevado a confrontar un sinfín de vericuetos teóricos y prácticos, todo esto con el propósito de volver a colocar al psicoanálisis en las vías de su causa original.

Lacan en 1957 haciendo alusión al ideal cientificista que claramente animaba el discurso de algunos psicoanalistas de la Asociación Psicoanalítica Internacional (IPA) y de la cual aún formaba parte, referirá que en el campo de lo humano el solo hecho de hablar de experiencia ya coloca la relación sujeto-objeto en una dimensión subjetiva. Este pensamiento le condujo un año más tarde a escribir una fuerte crítica sobre aquellas "ciencias" que por su formulación teórico-práctica se asumen estudiosas de la subjetividad, citamos: “(...) lo único que hacen es interponerse entre nosotros y lo que designamos cuando hacemos intervenir la subjetividad en nuestra experiencia (...)" (Lacan, 1957-58/2010, p. 108), a renglón seguido enfatizará que el abordaje de lo subjetivo fuera del psicoanálisis desemboca en formas de objetivación de lo más vulgares.

De lo anterior es comprensible el interés surgido en esos años de parte del psicoanalista francés por establecer las bases necesarias que permitieran diferenciar la investigación psicoanalítica de otras. En ese mismo año en su escrito acerca de "Las formaciones del inconsciente" sostendrá que la noción de sujeto en el campo del psicoanálisis resulta incompatible con cualquier otra fórmula, planteando inclusive, que aquellas ideas que colocan la noción de sujeto como resultado de una inmanencia consciente no hacen más que sintetizar el espejismo objetivista que las funda (Lacan, 1957-58/2010). 
Para esa misma época propondrá al cogito Cartesiano como un punto de referencia importante en la construcción de las nociones sujetoobjeto dentro de la teoría freudiana, sin embargo, dejará por sentado la necesidad de establecer un distanciamiento que de testimonio de los avances teóricos de una praxis que se sostiene en el marco de los nuevos desafíos. En esta perspectiva para 1965 comunicará qué, el sujeto sobre el cual opera el psicoanálisis no puede ser sino el sujeto de la ciencia, aunque esto resulte paradójico, es ahí, donde debe hacerse una demarcación (Carvalho \& Guerra, 2013; Santos, 2014; Vorsatz, 2015). El problema a decir del autor ha sido la falta de capacidad por parte de los discursos objetivos, al no poder identificar que aquello que realmente falla en el objeto no está del lado de este, sino del lado del sujeto (Lacan, 1965-66).

Prudente y Ribeiro (2005) plantean que gracias a la subversión del cogito Cartesiano realizado por Lacan a favor del psicoanálisis, se ha podido construir una concepción de sujeto-objeto que le son propias. Si bien no se trató de nada nuevo a lo elaborado por Freud en la "interpretación de los sueños" el franqueamiento y delimitación que el psicoanalista francés le otorgó a la fórmula de Descartes permitió a su vez sostener con mayor fundamentación lógica, el rechazo de un saber inconsciente por parte de la ciencia moderna y que hoy sabemos es el objeto de estudio del psicoanálisis. Fue a la luz de estas elucidaciones que Lacan daría continuidad al "esfuerzo" freudiano de otorgarle al método psicoanalítico su lugar propio dentro de las ciencias.

La relectura emprendida por Lacan al cogito Cartesiano trajo consigo no solo la delimitación de concepciones que al pasar al campo del psicoanálisis expresan una realidad distinta, sino también la germinación de ideas tendientes a reformular conceptos incluso dentro del mismo psicoanálisis (Fernández, 1999; Prudente \& Ribeiro, 2005; Jardim \& Rojas, 2010; França, 2015; Gault, 2015).

Jacques Lacan al tiempo en que se pronunciaba y teorizaba con el propósito de superar el sentido convencional del binario sujeto-objeto de la teoría del conocimiento, evidentemente también dejaba entrever la dificultad teórica-práctica de aquellos que se hacían llamar los herederos de Freud, en este sentido demostró que la IPA no era más que un aparato gigantesco normalizador, lugar donde se podían elaborar las más severas confusiones metodológicas sin el menor dejo de discusión (Miller, 2011).

Esta serie de críticas que se agudizaban en la medida en que el psicoanalista francés alejaba el dispositivo freudiano de nociones tergiversadas, le costó su "excomunión" de la IPA en 1963, sin embargo, era el precio necesario a condición de salir del oscurantismo al cual había sido sometido el método psicoanalítico durante varias décadas.

Gracias al recorrido realizado por Lacan a lo largo de su enseñanza se pudo hacer dentro del campo psicoanalítico una separación entre su objeto de estudio y aquel abordado por la ciencia. Para Rabinovich (2003) el atributo sensible del objeto al que puede aspirar la ciencia esconde en su núcleo mismo la dimensión inasimilable de éste.

\section{El objeto del psicoanálisis}

Las diferencias entre el psicoanálisis y la racionalidad científica clásica sobre el binomio sujeto-objeto, más la tesis freudiana que proponía a su dispositivo "como un método de investigación de procesos anímicos difícilmente accesibles por otras vías" (Freud, 1923-25/1992, p. 231) al parecer resultaron ser demasiado para los psicoanalistas "herederos" de Freud, de tal modo que después de su muerte el círculo intelectual más cercano a él gestó una serie de confusiones conceptuales que se tradujeron también en dificultades metodológicas. Esta razón condujo a Lacan a buscar desde su propia trinchera un retorno a la causa freudiana original, en una conferencia dictada en Milán declarará el objetivo de su enseñanza de la siguiente manera: "Desde hace tiempo hacemos un esfuerzo por fundar una práctica del discurso que se sostenga" (Lacan, 1973/1978, p. 3, [énfasis propio]). En este mismo sentido Sauval en 1999, propondrá asumir el compromiso constante de conservar dentro del dispositivo psicoanalítico la singularidad de su praxis.

Durante esos años el psicoanalista francés se pronunciará fuertemente sobre aquellas "disciplinas" estudiosas de la subjetividad que han conservado en el marco de su "investigación" una práctica contemplativa de sus objetos, dentro de éstas también incluirá a un tipo de "psicoanálisis" que después de Freud sostuvo su quehacer en el estudio de las funciones Yoicas, es decir, una racionalidad fronteriza cuya superficie es imposible fijar (Bayón, 2007), desconociendo por completo 
la hiancia que provoca la función simbólica en la que se engrana toda esa historia, a modo de acentuación dirá lo siguiente: “(...) esa contemplación del mundo sería identificada de alguna manera con lo que es (...) una gran ilusión (...)" (Lacan, 1973/1978).

Resulta obvio que los comentarios realizados por Lacan estaban dirigidos a poner sobre la mesa los intentos fallidos por parte de la IPA de continuar con la transmisión de la investigación de los fenómenos psíquicos. Esta teorización socavaba indudablemente la tradición de una asociación psicoanalítica que durante largos años se había asumido como la responsable de formar teórica y metodológicamente a psicoanalistas para el ejercicio de la práctica clínica.

Cabe mencionar que para la época en que Lacan realizaba estas precisiones, sus desencuentros con las ideas generadas dentro de la IPA ya eran añejas, así, tenemos que durante el XIV Congreso de esta asociación llevada a cabo en Marienbad, el 03 de agosto de 1936 mientras Ernest Jones fungía como su presidente, este le impidió concluir a Lacan su primera participación internacional sobre El estadio del espejo. Dicha conferencia contenía el resultado de sus investigaciones propiamente psicoanalíticas que como era de entenderse contradecían las proposiciones sostenidas por la IPA.

En 1966 tres años después de su separación formal de esta Asociación internacional dentro de su presentación escrita titulada: "Nuestros antecedentes" volverá al pronunciamiento realizado en El estadio del espejo refiriendo lo siguiente: “(...) su invención nos colocaba en el interior de una resistencia teórica y metodológica que, aunque constituía un problema que después fue más fuerte, se hallaba, preciso es decirlo lejos de ser percibido" (Lacan, 1966/2005, p. 60). Dos años antes, en 1964 ya había dictado una serie de conferencias que, a decir por él, le permitieron volver sobre los fundamentos freudianos originales, los mismos que durante largos años se encontraran en contradicción (Lacan, 1964/2009; Druet, 2014).

Los dos cuestionamientos que dirigirán sus reflexiones durante esta época serán, por un lado, los fundamentos de la praxis psicoanalítica y por el otro, si el psicoanálisis es capaz de inscribirse en los registros de la "ciencia"; en lo que respecta a la primera cuestión, ésta tendrá su más formal peso derivado de la posición de sus colegas e incluso sus analizantes al momento de "negociar" su salida de la IPA, se trata en otras palabras, de colocar la "investigación psicoanalítica" en el marco de una práctica ética que permita a quién aborda lo Inconsciente de abstenerse de juicios morales e ideales yoicos (Lacan, 1964/2009; Hochman, 2012); en referencia a la segunda interrogante Lacan precisará que en términos de investigación existen al menos dos terrenos perfectamente deslindarles: el dominio de donde se busca y el dominio de donde se encuentra (Miller, 2011), el psicoanálisis se ubica en los terrenos del encuentro con un saber que no se sabe (Freud, 1915-16/1992), en otras palabras, el objeto del psicoanálisis tendríamos que situarlo en todo caso, fuera del dominio del propio sujeto, en el punto mismo de su división subjetiva, por donde especialmente alcanza su estructuración (Lacan, 1965-66).

Continuando con su esfuerzo por delimitar el objeto del psicoanálisis Lacan argumentará que, desde el surgimiento de la "ciencia moderna" esta ha mantenido pendiente algo por dilucidar del estatuto de su objeto, gracias a ello Freud localizaría en el sujeto un "saber reprimido", es decir un objeto cuya cualidad es la de ser inaprensible.

Para Zaidel (2010) en el campo de las ciencias los objetos pueden ser definidos y concretos si se le aplica a la realidad una red simbólica, sin embargo, en esta operación inevitablemente algo del objeto queda fuera. Rouse en 2013 señala que, durante el dominio del saber "metafísico" se asumía entre el sujeto y el objeto una armonía, no obstante, esta correspondencia "idealizada" se vio fracturada al momento de asumir la ciencia moderna la responsabilidad de construir su objeto, movimiento que subraya Lacan como el carácter específicamente subjetivo de la estructura científica, que pudo haber permitido al menos "levemente" entrever la inverosímil complementariedad entre sujeto-objeto (Lacan, 1965-66; Rouse, 2013).

Esta diferencia que coloca al objeto en una imposibilidad de aprehensión se torna para la ciencia en una consideración secundaria frente a la premisa de introducir representaciones de este (Zaidel, 2010). Se trata para decirlo de otra manera:

Si el objeto de la ciencia les apasiona es porque ahí dentro, oculto en él está el objeto del deseo(...) Este objeto privilegiado que culmina para cada cual, en aquella frontera, en aquel punto límite que les he enseñado a 
considerar como la metonimia del discurso inconsciente. (Lacan, 1960-61/1987, p. 171).

Asumiendo estas elaboraciones lacanianas podemos sostener que la ciencia mediante el mecanismo de construcción de significantes forcluye la verdad sobre su objeto, liberando al mismo tiempo al sujeto del peso que constituye su división subjetiva (Sprott, 2013), este proceso trae consigo el encadenamiento de una serie de "experiencias humanas" que conforma para la llamada ciencia el "conocimiento" sobre su objeto, desechando por otro lado la "causa" que en todo caso es su condición más radical, por el contrario, el psicoanálisis desde su creación se distingue por asumir esta "división" como primordial, arrastrando las consecuencias de esa "verdad".

En suma, aquello que desde un principio situó a la investigación psicoanalítica en las vías de la producción de "un saber sobre el objeto como causa de una verdad" que constituye la posición de cada sujeto, en el campo de la ciencia ha permanecido rechazada (Bustamante, 2009), al momento de perfilarse sobre un ideal que busca sustraer de los objetos del mundo un conocimiento absoluto. En este sentido lo que la ciencia pierde de vista es que aquello rechazado en lo simbólico reaparece en lo real (Lacan, 1959-60/2010), provocando que el conocimiento que se produce en su campo no pueda ser más que semblante en tanto representación y efecto de una "falta" de objeto en lo Real.

\section{Conclusiones}

La validez y objetividad del dispositivo psicoanalítico en su conjunto, tanto en teoría como en práctica ha sido duramente criticado a lo largo de su historia, no obstante y gracias a sus efectos la mayor parte de sus postulados aún siguen vigentes.

Las primeras ideas freudianas sobre los fenómenos que atañen al cuerpo fueron inaceptadas por sus contemporáneos, inclusive antes de acuñar el término psicoanálisis. Con el tiempo el blanco de los desprecios se generalizó hacia su persona, sin embargo estoicamente asumió las consecuencias de su causa, al precio de hacer emerger la verdad insoslayable de la constitución humana y que la ciencia exacta a modo, había rechazado.

Esta nueva fórmula además de romper abruptamente con el pensamiento de su época, colocó sobre la mesa una nueva noción de objeto, revistiéndolo de aquellas cualidades que el discurso de la ciencia moderna había despojado hasta entonces. Sobre este nuevo modo de pensar la realidad de los objetos y como resultado de su práctica clínica, el padre del psicoanálisis llegará a establecer con el tiempo una nueva causalidad lógica, a saber, que de nuestra posición de sujetos, somos siempre responsables.

Por otro lado, la claridad teórico-práctica de Freud y la posición ética con la cual se condujo en cada momento de la historia de su movimiento no deja ninguna duda de que sus descubrimientos fueron por demás sistemáticos y rigurosos, sin embargo, al desarrollarse ésta investigación en la singularidad de cada caso esta no admite comparación con una práctica investigativa donde el objeto que se estudia puede ser aprehensible, observable y cuantificable.

La decisión de Freud por colocar sus descubrimiento al servicio de la ciencia natural y que desde el "Proyecto..." mostraba claros indicios de ser sofocada, pudiendo anticipar que el destino de esta nueva disciplina no se encontraba en los terrenos de la cientificidad exacta, no obstante como lo dirá Lacan años después, el psicoanálisis no pudo haber visto la luz sin el surgimiento del sujeto Cartesiano, empero a condición de su subversión.

En este sentido, el lugar que guarda el psicoanálisis entre las ciencias puede ser comprensible a la luz de lo que éste produce como efecto de su práctica.

No fue azar que Lacan después de la subversión del cogito Cartesiano asumiera el compromiso adquirido por Freud de delimitar el campo de investigación psicoanalítica. La determinación de Lacan de volver a encauzar el deseo de Freud y que al mismo tiempo atizo su expulsión de la Asociación Psicoanalítica Internacional trajo como consecuencia una claridad plausible, al colocar en el centro del estudio de la subjetividad humana, los efectos que surgen al momento de poner en juego la relación sujeto-objeto.

Dicho lo anterior, allí donde la ciencia sostiene que mediante un control racional del sujeto sobre el objeto se propicia la condición necesaria para posteriormente elaborar un conocimiento exacto sobre éste, el psicoanálisis asumirá, que el objeto (también sujeto) no puede ser sometido a esos controles y por lo tanto, el conocimiento sobre ellos únicamente puede ser parcial, dicho de otra manera, contrariamente a la soberbia racionalidad 
científica de circunscribir al investigador y al objeto a una mecánica orquestada a fin de congelar sus cualidades, el psicoanálisis ha colocado como ilusoria esta supuesta autoridad de control y peor aun históricamente ha señalado que todo aquello que atañe al investigador trae efectos al momento de abordar su objeto.

Por otro lado, una de las críticas más fuertes que propuso Lacan para delimitar con mayor claridad el distanciamiento que guarda el psicoanálisis con respecto a la ciencia moderna fue la tesis de que en su objeto esta última esconde el núcleo mismo la verdad que lo estructura y, por lo tanto, en su abordaje siempre queda algo que se le escapa.

Esta observación coloca al objeto del psicoanálisis como "inaprensible" para la investigación científica. La fórmula que desde Freud se ha procurado construir, con la finalidad de establecer un método capaz de hacer emerger eso que para el propio sujeto aparece como -excluido interiormente- y que mediante la palabra puede alcanzar su aspecto de causa material, pareciera que a la luz de nuestros días no ha alcanzado mayor determinación. En este sentido, la responsabilidad asumida por Freud y Lacan de otorgarle al psicoanálisis un lugar de extimidad con respecto a la llamada ciencia, no ha podido conseguirse del todo, esto se confirma al encontrarnos hoy en día con prácticas denominadas psicoanalíticas con claras aspiraciones objetivistas y carentes de rigurosidad metodológica. 


\section{Referencias}

Antonello, D. \& Oliveira, R. (2011). A repetição e o Projeto de 1895: gérmen de um conceito. Revista Latinoamericana de Psicopatologia Fundamental, 14(2), 237-251. doi https://dx.doi. org/10.1590/S1415-47142011000200003

Ávila, L. (2005). Transferência e complexo de édipo, na obra de Freud: notas sobre os destinos da transferência. Psicologia: Reflexão e Crítica, 18(1), 143-149. doi https://dx.doi.org/10.1590/ S0102-79722005000100019

Armengol, R. (2007). Validez y vigencia del psicoanálisis de Freud. Revista Medicina Clínica, 128(16), 619-622. doi https:// doi.org/10.1157/13101751

Barcellos, B. \& Magdalen, R. (2012). Saturação teórica em pesquisas qualitativas: contribuições psicanalíticas. Psicologia em Estudo, 17(1), 63-71. Extraido de http://www.scielo.br/pdf/ pe/v17n1/v17n1a07.pdf

Bayón, F. (2007). Freud y la crisis del lenguaje moderno en la Viena fin de siglo: Broch, Hofmannsthal, Kraus. Revista ARBOR, Ciencia, Pensamiento y Cultura, 183(723), 135-154. doi https:// doi.org/10.3989/arbor.2007.i723.86

Berrios, G. (2011). Psychiatry and its objects. Revista de Psiquiatría y Salud Mental, 4(4), 179-82. doi https://doi:10.1016/j. rpsm.2011.09.001

Blas, H., Azcona, M., \& Ortiz O. (2013). La noción de causalidad en Sigmund Freud. Límite. Revista Interdisciplinaria de Filosofía y Psicología, 8(27), 59-74. Extraido de http://www. redalyc.org/pdf/836/83628140004.pdf

Breuer, J. \& Freud, S. (1893-95/1992). Estudios sobre la histeria (Obras completas tomo II). Buenos Aires: Editorial Amorrortu.

Brooks, B. (2017). La objetividad de las ciencias naturales: un meta-análisis de la investigación sobre el lago de Pátzcuaro. Revista Teoría y Crítica de la Psicología, 9, 98-123. Extraido de http://www.teocripsi.com/ojs/index.php/TCP/article/view/162/169

Brousse, M. (abril, 2012). Descartes con Lacan. Conferencia pronunciada previo al Primer Coloquio-Seminario de la Orientación Lacaniana, ciudad de la Plata, Argentina. Extraido de http://www.eol-laplata.org/blog/index.php/ conferencia-de-marie-helene-brousse-descartes-con-lacan/

Boonen, C. \& Calazans, R. (2017). A noção de sujeito do inconsciente como situação imanejada. Tempo psicanalitico, 49(1), 98-122. Extraido de http://pepsic.bvsalud.org/scielo. php?script=sci_arttext\&pid=S0101-48382017000100006\&ln $\mathrm{g}=\mathrm{es} \& \mathrm{t} \operatorname{lng}=\mathrm{pt}$.

Bustamante, G. (2009). Investigación, psicoanálisis, educación Parte I: posición metodológica y epistemológica. Magis. Revista Internacional de Investigación en Educación, 1(2), 249-260. Extraido de http://revistas.javeriana.edu.co/index.php/MAGIS/ article/view/3382

Canale, F. (2011). La anomalía histérica en el origen del psicoanálisis. Revista Colombiana de Filosofía de la Ciencia, 11(22), 83-88. Extraido de http://www.redalyc.org/pdf/414/41421595007.pdf

Carvalho, G. \& Guerra, A. (2013). Notas sobre o sujeito da psicanálise. Tempo psicanalitico, 45(1), 123-134. Extraido de http://pepsic.bvsalud.org/scielo.php?script=sci_ arttext\&pid=S0101-48382013000100009\&lng=es\&tlng= pt.

Chavarría, M. (2011). La dicotomía cuantitativo/cualitativo: falsos dilemas en investigación social. Actualidades en Psicología, 25,
1-35. Extraido de http://pepsic.bvsalud.org/scielo.php?script=sci_ar ttext\&pid=S0258-64442011000100001

Coccoz, V. (2012). El psicoanálisis: una ética para el siglo XXI. Conferencia del Instituto del Campo Freudiano de Granada, Granada, España.

Cupani, A. (2011). Acerca de la vigencia del ideal de objetividad científica. Scientiae Studia, 9(3), 501-525. doi http://dx.doi. org/10.1590/S1678-31662011000300004

Daston, L. \& Galison, P. (2007). Objetividad. New York: Zone Books.

Druet, A. (2014). La psiquiatría española y Jacques Lacan antes de 1975. Asclepio, 66(1), 1-36. doi http://dx.doi.org/10.3989/ asclepio.2014.10

Emilce, F. (2015). Razones y causas en el psicoanálisis freudiano. Eidos, 23, 135-156. doi https://dx.doi.org/10.14482/eidos.23.190

Fernández, P. (1999). Epistemología y Psicoanálisis. Revista Cinta de Moebio, 5. Extraido de https://www.moebio.uchile. $\mathrm{cl} / 05 /$ psicoanalisis.html

França, O. (2015). Uma metodología para a psicanálise. Psicologia Clínica, 27(1), 195-211. doi https://dx.doi. org/10.1590/0103-56652015000100011

França, O. (2016). Considerações psicanalíticas sobre dificuldades da metodologia nas ciências que lidam com sujeitos. Psicologia em Estudo, 21(4), 593-602. doi http://dx.doi.org/10.4025/psicolestud.v21i4.32342

Freud, S. (1893-99/1992). Primeras publicaciones psicoanalíticas (Obras completas, tomo III). Buenos Aires: Editorial Amorrortu.

Freud, S. (1900/1992). La interpretación de los sueños (Obras completas, tomo IV). Buenos Aires: Editorial Amorrortu.

Freud, S. (1914-16/1992). Contribución a la historia del movimiento psicoanalítico. Trabajos sobre metapsicología y otras obras (Obras completas, tomo XIV). Buenos Aires: Editorial Amorrortu.

Freud, S. (1915-16/1992). Conferencias de introducción al psicoanálisis (Partes I y II) (Obras completas, tomo XIV). Buenos Aires: Editorial Amorrortu.

Freud, S. (1917-19/1992). De la historia de una neurosis infantil (el hombre de los lobos) (Obras completas, tomo XVII). Buenos Aires: Editorial Amorrortu.

Freud, S. (1920-22/1992). Más allá del principio del placer. Psicología de las masas y análisis del yo y otras obras (Obras completas, tomo XVIII). Buenos Aires: Editorial Amorrortu.

Freud, S. (1923-25/1992). El yo y el ello y otras obras (Obras completas, tomo XIX). Buenos Aires: Editorial Amorrortu.

Freud, S. (1937-39/1992). Moisés y la religión monoteísta. Esquema de psicoanálisis y otras obras (Obras completas, tomo XXIII). Buenos Aires: Editorial Amorrortu.

Fuks, B. (2017). "Pergunte ao cavalo!": sobre o inconsciente freudiano. Tempo psicanalitico, 49(1), 123-138. Extraido de http://pepsic.bvsalud.org/scielo.php?script=sci_ arttext\&pid=S0101-48382017000100007\&lng=es\&tlng= pt.

Fulgencio, L. (2004). O Projeto como uma metáfora biológica dos processos psíquicos. Psicologia USP, 15(3), 117-135. doi https://dx.doi.org/10.1590/S0103-65642004000200007 
Gammelgaard, J. (2015). The talking cure: psychoanalysis and the ambiguity of language. The Scandinavian Psychoanalytic Review, 38(2), 86-93. doi https://doi.org/10.1080/01062301.2 015.1096168

Garcia-Mendes, E. (1996). Freud e a fisiologia. Estudos Avançados, 10(27), 79-93. doi https://dx.doi.org/10.1590/ S0103-40141996000200004

Gargiulo, M. (2014). La crítica de Paul Karl Feyerabend al modelo neo-positivista del significado. Revista ARBOR, Ciencia, Pensamiento y Cultura, 190(769), 1-18. doi http://dx.doi. org/10.3989/arbor.2014.769n5007

Gault, J. (2015). O nascimento da ciência moderna. Uma leitura de "A ciência e a verdade". Arquivos Brasileiros de Psicologia, 67(2), 156-161. Extraido de http://pepsic.bvsalud.org/scielo. php?script=sci_arttext\&pid=S1809-52672015000200012

Gildersleeve, M. (2016). Method in the Madness: Hysteria and the Will to Power. Revista Social Sciences, 5(3), 1-29. doi http:// dx.10.3390/socsci5030029

González, M., Hernández, A., \& Antonio A. (2014). Positivismo, dialéctica materialista y fenomenología: tres enfoques filosóficos del método científico y la investigación educativa. Revista Electrónica Actualidades Investigativas en Educación, 1(20). Extraido de http://www.redalyc.org/articulo.oa?id=44732048021

González, A., Domínguez, M., Fabre, O., \& Cubero, A. (2010). La influencia de Descartes en el desarrollo del método anatomoclínico. Neurología, 25(6), 374-377. doi https://doi.org/10.1016/j. nrl.2009.12.011

Gutiérrez, J. (2004). El método de investigación psicoanalítico y el proceso conversacional en la investigación social cualitativa. Empiria, Revista de Metodología de Ciencias Sociales, 7, 77-98. Extraido de http://www.revistas.uned.es/index.php/empiria/ article/download/968/887

Habermas, J. (1968/1982). Conocimiento e interés. Madrid: Editorial Taurus.

Harada, E. (2006). Observación, teorías y valores a la luz de la filosofía de Popper. Ciencia Ergo Sum, 13(2), 200-210. Extraido de http://www.redalyc.org/articulo.oa?id=10413212

Henríquez, R. (2010). Vigencia del Proyecto de una psicología para neurólogos. Anales del Seminario de Historia de la Filosofía, 27, 277-294. Extraido de http://www.redalyc.org/ articulo.oa? $\mathrm{id}=361133110011$

Hochman, P. (2012). El sujeto: Una noción ética. Revista Tesis psicológica, 7(12), 178-187. Extraido de http://publicaciones.libertadores.edu.co/index.php/TesisPsicologica/article/view/255/250

Jardim, L. \& Rojas, M. (2010). Investigación psicoanalítica en la universidad. Estudos de Psicologia (Campinas), 27(4), 529536. doi https://dx.doi.org/10.1590/S0103-166X2010000400010

Lacan, J. (1954-55/2008). El Yo en Teoría de Freud y en la Técnica psicoanalítica (El seminario de Jacques Lacan, libro 2). Buenos Aires: Editorial Paidós.

Lacan, J. (1957-58/2010). Las formaciones del inconsciente (El seminario de Jacques Lacan, libro 5). Buenos Aires: Editorial Paidós.

Lacan, J. (1960-61/1987). La transferencia (El seminario de Jacques Lacan, libro 8). Buenos Aires: Editorial Paidós.

Lacan, J. (1964/2009). Los cuatro conceptos fundamentales del psicoanálisis (El seminario de Jacques Lacan, libro 11). Buenos Aires: Editorial Paidós.
Lacan, J. (1965-66). El objeto del psicoanálisis (El seminario de Jacques Lacan, libro 13). Inédito.

Lacan, J. (1966/2005). Escritos 1. Ciudad de México: Editorial Siglo XXI.

Lacan, J. (1975/2011). Escritos 2. Ciudad de México: Editorial Siglo XXI

Lacan, J. (1973/1978). Lacan en Italia 1953-1978. Milán: La Salamandra (pp. 58-77). Extraido de http://ecole-lacanienne.net/ wp-content/uploads/2016/04/Lacan_Milan_3-fevrier_1973.pdf

Legendre, P. (2008). El tajo. Discurso a jóvenes estudiantes sobre la ciencia y la ignorancia. Buenos Aires: Editorial Amorrortu.

Lindenmeyer, C. (2012). Qual é o estatuto do corpo na psicanálise? Tempo psicanalitico, 44(2), 341-359. Extraido de http://pepsic.bvsalud.org/scielo.php?script=sci_ arttext\&pid=S0101-48382012000200006\&lng=es\&tlng= pt.

Lutereau, L. (2010). Actualidad de la metapsicología freudiana. Una revisión de sus problemas y una evaluación de sus soluciones. Revista Desde jardín Freud, 10, 99-108. Extraido de https://revistas.unal.edu.co/index.php/jardin/article/view/19829

Mannoni, M. (2002). Un saber que no se sabe. La experiencia analítica. Barcelona: Editorial Gedisa.

Masson, J. (1985) Sigmund Freud Cartas a Wilhelm Fliess (1887-1904). Buenos Aires: Editorial Amorrortu.

Méndez, E. (2000). El desarrollo de la ciencia. Un enfoque epistemológico. Espacio Abierto, 9(4), 505-534. Extraido de http://www.redalyc.org/articulo.oa?id=122904

Miller, J. (2011). La vida de Lacan. Buenos Aires: Grama Ediciones

Ovejero, A. (1995). Reseña de "Freud: una vida de nuestro tiempo" de Peter Gay. Psicothema, 7(2), 453-460. Extraido de http://www.psicothema.com/psicothema.asp?id=991

Pezo, M. (octubre, 2006). Freud y el positivismo de su época: ¿El psicoanálisis una ciencia positivista? Trabajo presentado en el XXVI Congreso Latinoamericano de psicoanálisis, "El legado de Freud a 150 Años de su nacimiento", Lima, Perú. Extraido de http://fepal.org/images/2006invest/pezo_del_pino.pdf

Prudente, R. \& Ribeiro, M. (2005). Psicanálise e ciência. Psicologia: Ciência e Profissão, 25(1), 58-69. doi https://dx.doi. org/10.1590/S1414-98932005000100006

Rancaño, P. (2011). L'Hôpital Pitie-Salpêtrière en París. Revista Científica de la Sociedad Española de Enfermería Neurológica, 0(34), 25-7. Extraido de http://www.elsevier. es/es-revista-revista-cientifica-sociedad-espanola-enfermeria319-articulo-1-hopital-pitie-salpetriere-paris-X201352461121 0723?referer=buscador

Ramírez, M. (2002). Freud y el positivismo científico. Revista Documenta laboris (serie de trabajos y estudios de investigación de la escuela de graduados), 6, 95-114. Extraido de https:// dialnet.unirioja.es/servlet/autor?codigo $=331269$

Rabinovich, D. (2003). El concepto de objeto en la teoría psicoanalítica. Buenos Aires: Manantial.

Rodríguez, P. (2010). El positivismo y el racionalismo no han muerto. Educere, 14(48), 63-71. Extraido de http://www.redalyc. org/articulo.oa?id=35616720007

Rouse, H. (2013). El objeto del conocimiento precientífico; el objeto de la ciencia; y el objeto del psicoanálisis. NODVS l'aperiòdic virtual de la Secció Clínica de Barcelona, 41, 
Extraido de http://www.scb-icf.net/nodus/contingut/article. php?art $=504 \&$ rev $=60 \&$ pub $=1$

Rubistein, A. (1997). Investigar en Psicoanálisis. Revista El Caldero de la Escuela, 50, s/n. Buenos Aires: Grama ediciones.

Rudge, A. (2012). Destinos do método clínico na contemporaneidade. Revista Latinoamericana de Psicopatologia Fundamental, 15(3), 512-523. doi https://dx.doi.org/10.1590/ S1415-47142012000300005

Rudge, A. (2016). Sonhos traumáticos na clínica psicanalítica. Revista Latinoamericana de Psicopatologia Fundamental, 19(4), 603615. doi https://dx.doi.org/10.1590/1415-4714.2016v19n4p603.2

Ruiz, E. (2009). El psicoanálisis y el saber acerca de la subjetividad. Revista Espiral, Estudios sobre Estado y Sociedad, 16(46), 37-58. Extraido de http://www.scielo.org.mx/scielo. php?script=sci_arttext $\&$ pid $=$ S1665-05652009000100002 \&ln $\mathrm{g}=\mathrm{es} \& \mathrm{nrm}=\mathrm{iso} \&$ tlng=es

Sánchez, A., Sánchez, P., \& Sánchez, F. (2005). El psicoanálisis: ¿Qué tipo de ciencia es? Revista de la Asociación Española de Neuropsiquiatría, XXV(96), 93-111. Extraido de http://www. aen.es/web/docs/RevAEN96.pdf

Sánchez-Domínguez, J. (2016a). Estudio de caso: una manera de investigar en psicoanálisis. Ajayu, Órgano de Difusión Científica del Departamento de Psicología UCBSP, 14(1), 7-22. Extraido de http://www.scielo.org.bo/scielo. php?script=sci_arttext\&pid=S2077-21612016000100002\&ln $\mathrm{g}=\mathrm{es} \& \mathrm{t} \operatorname{lng}=\mathrm{es}$.

Sánchez-Domínguez, J. (2016b). Los límites de la racionalidad a propósito de las identidades sexuales: el caso de Herculine Barbin. Limite. Revista Interdisciplinaria de Filosofía y Psicología, 11(36), 60-73. Extraido de http://limite.uta.cl/index.php/limite/ article/view/194

Santos, T. (2014). La pratique psychanalytique et sa jouis-science. Revista Latinoamericana de Psicopatologia Fundamental, 17(2), 218-233. doi https://dx.doi.org/10.1590/1984-0381v17n2a06
Sauval, M. (1999). La "formación" del analista. Los impasses de Freud y la IPA. Acheronta, Revista de psicoanálisis y cultura, 9. Extraido de http://www.acheronta.org/acheronta9/formacion.htm

Simanke, R. \& Caropreso, F. (2005). O conceito de consciência no Projeto de uma psicologia de Freud e suas implicações metapsicológicas. Trans/Form/Ação, 28(1), 85-108. doi https:// dx.doi.org/10.1590/S0101-31732005000100005

Sprott, F. (2013). "Last Seen Alive": Lacan, Louise Bell and I in a Haunted House. Rupkatha, Journal on Interdisciplinary Studies in Humanities, 5(2), 301-313. Extraido de http://rupkatha. com/lacan-louise-bell/

Tessier, H. (2012). Métapsychologie, épistémologie et éthique de la clinique psychanalytique. Psicologia em Estudo, 17(3), 373381. doi http://dx.doi.org/10.1590/S1413-73722012000300003.

Torrades, S. (2005). La naturaleza de los sueños. Revista Offarm, 9(24), 134-40. Extraido de http://www.elsevier.es/es-revista-offarm4-articulo-la-naturaleza-los-suenos-13079597?referer=buscador

Tubert, S. (2007). Fluctuat nec mergitur: el psicoanálisis en el siglo XX. Revista ARBOR, Ciencia, Pensamiento y Cultura, 183(723), 7-25. doi https://doi.org/10.3989/arbor.2007.i723.78

Vorsatz, I. (2015). O sujeito da psicanálise e o sujeito da ciência: Descartes, Freud e Lacan. Psicologia Clínica, 27(2), 249-273. Extraido de http://pepsic.bvsalud.org/scielo. php?script=sci_arttext\&pid=S0103-56652015000200013\&ln $\mathrm{g}=\mathrm{es} \& \mathrm{t} \operatorname{lng}=\mathrm{pt}$.

Villacañas, L. (2011). Marx y el ejemplo (sobre los límites de la racionalidad científica capitalista. ISEGORÍA. Revista de Filosofía Moral y Política, 44, 89-114. doi https://doi.org/10.3989/ isegoria.2011.i44.721

Zaidel, R. (2010). Ciencia y Psicoanálisis a partir del seminario XI. Laboratorio de criterios científicos, Universidad Popular JacquesLacan. Extraido de https://psicoanalisisyciencia.wordpress. com/2010/05/15/ciencia-y-psicoanalisis-a-partir-del-seminario-xi/ 


\section{Notas}

1 Entre 1882-1962, discípula y analizante predilecta de Freud; obtuvo las cartas y manuscritos que se originaron durante el periodo de correspondencia entre Freud y Fliess.

2 Si Freud sobreestimó el saber de Fliess sobre lo sexual, es justo para llegar a decir, a ubicar en el interior de esta relación, lo imposible de decir sobre el sexo, su falta (...)en la conversación todo está allí (...) sueño y síntoma (Lacan, 1954-55)
3 Médico y psiquiatra francés que formuló una teoría sistemática de la psicodinámica y acuñó el término de 'subconsciente'. En 1893 publica el estado mental de las histéricas. Trabajó en el hospital de la Salpêtrière con el neurólogo Jean Martin Charcot. En su disertación doctoral Janet formuló la existencia de fenómenos no conscientes en las histéricas.

4 Psiquiatra y psicoanalista francés, considerado uno de los más grandes intelectuales de nuestra época, realizó las mayores aportaciones al psicoanálisis después de Freud. 